\title{
QUANTITATIVE APPROACH TO ASSIST NEUROBLASTOMA ASSESSMENT BY MEASURING I-123 mIBG UPTAKE IN SCINTIGRAPHIC IMAGES
}

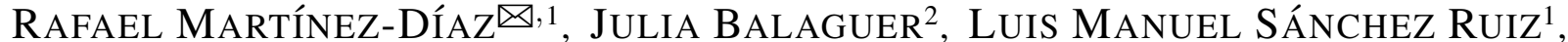 \\ Pilar Bello $^{3}$, Victoria Castel ${ }^{4}$, Antonio Rivas ${ }^{3}$, Adela Cañete ${ }^{2}$, Marina \\ FALGAS $^{3}$, JOSE LUIS LOAIZA ${ }^{3}$ AND GUILLERMO PERÍS-FAJARNÉS ${ }^{1}$
}

${ }^{1}$ Universitat Politècnica de València, Camino de Vera s/n, 46022 Valencia, Spain; ${ }^{2}$ Hospital La Fe, Paediatric Oncology Unit, Bulevar Sur s/n, 46026 Valencia, Spain; ${ }^{3}$ Hospital La Fe, Nuclear Medicine Unit, Bulevar Sur s/n, 46026 Valencia, Spain; ${ }^{4}$ Hospital La Fe, Health Research Institute, Bulevar Sur s/n, 46026 Valencia, Spain e-mail: rmartin@upv.es, julbagui@yahoo.es,lmsr@mat.upv.es, bello_pil@gva.es, castel_vic@gva.es, rivas_ant@gva.es, canyete_ade@gva.es, marinafalgas@gmail.com, loaiza_joseluis@ hotmail.com, gperis@upv.es (Received September 11, 2014; revised November 14, 2014; accepted January 27, 2015)

\begin{abstract}
Whole-body ${ }^{123}$ I-Metaiodobenzylguanidine (mIBG) scintigraphy is used as the primary image modality in neuroblastoma detection. It is the most sensitive and specific method for staging and response evaluation. Validated semi-quantitative scoring methods with low interobserver variability and high reproducibility have shown to be indispensable for the evaluation of response to therapy. However, low resolution, noise and acquisition difficulties, specially in children, make low definition scans. These facts increase observer dependent interpretations that limit assessment and complicate to put a scoring method succesfully into practice. It is essential to have an objective and reliable measure of response to test the activity of therapies. In this paper we propose the use of a quantitative observer-independent measurement of the strength of uptake to be used as an additional tool for assisting the International Society of Paediatric Oncology Europe Neuroblastoma Group (SIOPEN) semi-quantitative scoring method. This is the scoring method recommended by the SIOPEN Nuclear Medicine and Physics Committee, in collaborative work with the Children's Oncology Group, as the standard one for acquiring and reporting diagnostic paediatric mIBG scans across Europe. Our proposed method is based on the ratio between the amount of specific uptake at tumours and the amount of non-specific uptake at SIOPEN anatomical sectors which has shown to be constant in all the scans of the patients.
\end{abstract}

Keywords: image analysis, mIBG, neuroblastoma, quantitative assessment, scintigraphy.

\section{INTRODUCTION}

Neuroblastoma stands out among paediatric solid tumours because of its relative frequency. It is the third most common malignant solid tumour of childhood (Mueller et al., 2013) and it is metastatic in half of the patients at diagnosis, making accurate evaluation of metastatic sites challenging (Matthay et al., 2010). Anatomic imaging methods, such as computerized tomography (CT) and magnetic resonance imaging (MRI), are most useful for evaluation of the primary tumour mass and nearby involved lymph nodes. Functional imaging radioactive tracers, such as ${ }^{123} \mathrm{I}-$ mIBG, ${ }^{18} \mathrm{~F}-\mathrm{FDG}$, and ${ }^{99 m} \mathrm{Tc}-\mathrm{MDP}$, are used to assess the extent of disease and to search for distant metastases (Sharp et al., 2013).

${ }^{123}$ I-mIBG scintigraphy remains the most specific and sensible imaging modality of staging and response evaluation (virtually $100 \%$ of specificity) (Charron, 2013). It is essential in the management of neuroblastoma. mIBG enters neuroendocrine cells by an active uptake mechanism via the epinephrine transporter and is stored in the neurosecretory granules, resulting in a specific concentration in contrast to cells of other tissues.

The pathological distribution of ${ }^{123} \mathrm{I}-\mathrm{mIBG}$ is described as uptake in the primary tumour and in the metastatic sites (Bombardieri et al., 2010). High peaks of intensity are to be observed at location of ${ }^{123} \mathrm{I}$ mIBG avid tissues. This is called specific uptake. Nonspecific uptake is also observed at the whole body as a light homogeneous uptake pattern without peaks. It is due to the ${ }^{123} \mathrm{I}-\mathrm{mIBG}$ travelling in the bloodstream until it is excreted.

In order to evaluate the prognostic effects and quantify efficacy of therapy, numerous semiquantitative scores have emerged (Ady et al., 1995; Frappaz et al., 2000; Messina et al., 2006; Lewington et al., 2009; Yanik et al., 2013; Decarolis et al., 2013) although none of them has shown to be superior to the others for quantification of response to the 
treatment (Matthay et al., 2010). In 2004, nuclear medicine physicians and oncologists of the major cooperative groups from North America, Australia, New Zealand, Europe, China and Japan formed the International Neuroblastoma Risk Group (INRG). One of INRG's goals was to reach international consensus for standardized procedures for performing and scoring ${ }^{123} \mathrm{I}-\mathrm{mIBG}$ scans to minimize subjective assessments and improve the concordance between readers. The use of a semi-quantitative score has shown to be essential for evaluation of the efficacy of therapy (Brisse et al., 2011).

All these methods divide the skeleton into anatomical sectors, then give each sector an individual score for number of metastatic sites and strength of uptake. They have achieved good correlation with outcome. Intensity at uptake sites is usually scored as doubtful, definite, obvious or strong based upon the observer assessment in comparison to liver or other soft tissues.

But ${ }^{123} \mathrm{I}-\mathrm{mIBG}$ images have low definition, specially in paediatric neuroblastoma. Consecutive scans from the same patient may display variations of intensity. This is caused by a number of factors such as changes in the accumulation of radioisotopes during each examination, changes in the gamma camera, the volume of water intake, patient motion, the amount of urine excreted or the response to treatment. In addition, noise is also an important factor degrading scintigraphic images (Hannequin and Mas, 2002; Krom et al., 2013).

These factors limit the succesful application of the scoring methods, although they are diminished by strictly following the standarized guidelines for preparing the patient for the acquisition process defined in (Bombardieri et al., 2010). In Spain, La Fe Hospital is the center of reference for neuroblastoma and it is trying to institute the semi-quantitative scoring method recommended for the International Society of Paediatric Oncology Europe Neuroblastoma Group (SIOPEN; Lewington et al., 2009; 2011; Ladenstein et al., 2011) for neuroblastoma management. This method is being developing by the Nuclear Medicine and Physics Committee, in collaborative work with the Children's Oncology Group, for acquiring, reading and scoring paediatric mIBG scans. This collaborative group is setting the standards for the paediatric MIBG scans across Europe. The problems arise when image definition is poor. The disappearance or decreased intensity of a lesion could be very subjective in such cases and then intensity measurements show low concordance between different observers.

It is possible that response to therapy exists although it is too light to be visible for the human eye.
On the other hand, it is possible to have a light increase in uptake at a new metastatic site but not high enough to be clearly identified.

An objective measurement between specific uptake at the regions of interest (ROIs) such as tumours, metastases, etc. and non-specific uptake at a region of reference is needed. It could lead to a better identification and stratification of the cases.

First observer independent attempts to quantify uptake in ROIs in scintigraphies were made in (Maisey et al., 1973) where an uptake measurement of ${ }^{99 m} \mathrm{Tc}$ in thyroid studies based on the number of gamma photons detected (counts) was proposed. The main idea was to calculate the ratio between counts in ROI and the total body counts.

This idea is used in heart-to-mediastinal (H/M) uptake ratio derived from mIBG scintigraphy by providing a quantitative measurement of uptake with success to monitor response to medical treatment in cardiac sympathetic imaging (Inoue et al., 2013). The $\mathrm{H} / \mathrm{M}$ ratio is in widespread use, however, there are variations among publications from various institutions because of several factors (Chen et al., 2011; Bulten et al., 2012), especially, the way the ROIs are placed.

Computer-aided applications for automatic assessment of ${ }^{99 m} \mathrm{Tc}$ bone scintigraphic images have been developed as well. In them, brightness is used with success for feature identification. For instance, in Yin and Chiu (2004), local maximum of brightness in bones and its asymmetries are used to detect abnormalities in skeleton in ${ }^{99 m} \mathrm{Tc}$ whole body bone scintigraphies.

Later in Jia-Yann et al. (2007), a set of algorithms was also proposed for segmentation and lesion grey level determination in ${ }^{99 m} \mathrm{Tc}$ whole body bone scintigraphies. In this work, after smoothing noise and segmenting skeleton, the lesion grey level is used as an evidence of the existence of uptake. They used a set of previous measurements of pixel's intensity mean and standard deviation at different body parts in a set of 100 patients to create a grey level of reference. Then, any ROI could be measured in another patient and compared with the estimated normal grey level. The larger the standard deviation value of grey level is at a given region, the greater the possibility of bone lesion existence is.

${ }^{99 m} \mathrm{Tc}$ and ${ }^{123} \mathrm{I}-\mathrm{mIBG}$ are similar scintigraphic procedures and in both brightness is the primary tool used for abnormal region identification. Nevertheless, images obtained in ${ }^{99 m} \mathrm{Tc}$ scans are very different from ${ }^{123}$ I-mIBG due to a combination of diverse factors, 
the most important of which being that different radiopharmaceuticals have different biodistributions. For instance, previous work on bone segmentation with ${ }^{99 m} \mathrm{Tc}$ bone scintigrams in Sajn et al. (2005) based on detecting edges is not applicable as a general rule in paediatric neuroblastoma ${ }^{123} \mathrm{I}$-mIBG scintigraphies because bones are not always visible. Skeletal uptake is only visible in neuroblastoma in those patients with bone metastases. Thus, a quantitative measurement method that works even when no bones are visible in scan is required.

This brings out that application of techniques to improve image quality such as image enhancement based on histogram with successful application in detection of lesions in ${ }^{99 m} \mathrm{Tc}$ scintigraphies Jeong et al. (2011) seems not being useful in ${ }^{123} \mathrm{I}-\mathrm{mIBG}$ of children in neuroblastoma detection. In Wesolowski et al. (2005), although the Poisson noise level was reduced, nuclear medicine physicians preferred original (not processed) planar images rather than the processed version due the blur effect on features and general image degradation.

As a result of the above, the algorithms successfully used in ${ }^{99 m} \mathrm{Tc}$ are not directly applicable in ${ }^{123} \mathrm{I}$-mIBG. Nevertheless, the use of pixel's intensity mean and standard deviation at different body parts is also useful in ${ }^{123} \mathrm{I}-\mathrm{mIBG}$. In Martínez-Díaz et al. (2013), an observer independent method for measuring the uptake level in any body region was introduced. The technique enables the measurement of the relative amount of uptake with a quantitative unit based on the ratio between uptake at neuroblastoma tumours and metastases and the rest of the tissues.

In this paper, we analyse the use of this observer independent computerized procedure for the measurement of the strength of uptake as an additional tool to assist the SIOPEN semi-quantitative scoring method. Our approach is based on the ratio between the specific uptake at tumours or metastases and the non-specific constant uptake threshold of the rest of tissues at the SIOPEN body sector where a tumour is located. Regional comparison of uptake at tumour to uptake at predefined areas, such as the SIOPEN anatomical sectors, instead of the comparison to the local lesion site facilitates the repetitiveness of the process as it sets a fixed point of reference that helps in eliminating observer dependent variabilities.

This paper is structured as follows. In section Materials and Methods, we detail the proposed application of the measurement method defined in Martínez-Díaz et al. (2013) in the anatomical division defined by the SIOPEN semi-quantitative scoring method (Lewington et al., 2009; 2011; Ladenstein et al., 2011). Experimental results are provided in section Results, followed by discussion of the results in section Discussion and conclusions being drawn in section Conclusions.

\section{MATERIALS AND METHODS}

The experimental set contains twenty-seven ${ }^{123} \mathrm{I}$ mIBG scintigraphies of 10 patients under 14 years old, labeled $P A_{1}$ to $P A_{10}$, suffering from neuroblastoma. Images were acquired at $\mathrm{La} \mathrm{Fe}$ Hospital by its Nuclear Medicine Unit following the standardized guidelines described in (Bombardieri et al., 2010) after a slow intravenous injection of ${ }^{123}$ I-mIBG (Dose: $100 \mu \mathrm{Ci} / \mathrm{Kg}$ ) and using different scintillation cameras set to a photopeak equal to the principal gamma photon emitted, that is, of $159 \mathrm{keV}$. A minimum of 250000 and a maximum of 500000 photon counts were obtained for each anterior and posterior views. The use of these scintigraphies for this study was approved by the La Fe Hospital's Biomedical Research Ethics Committee.

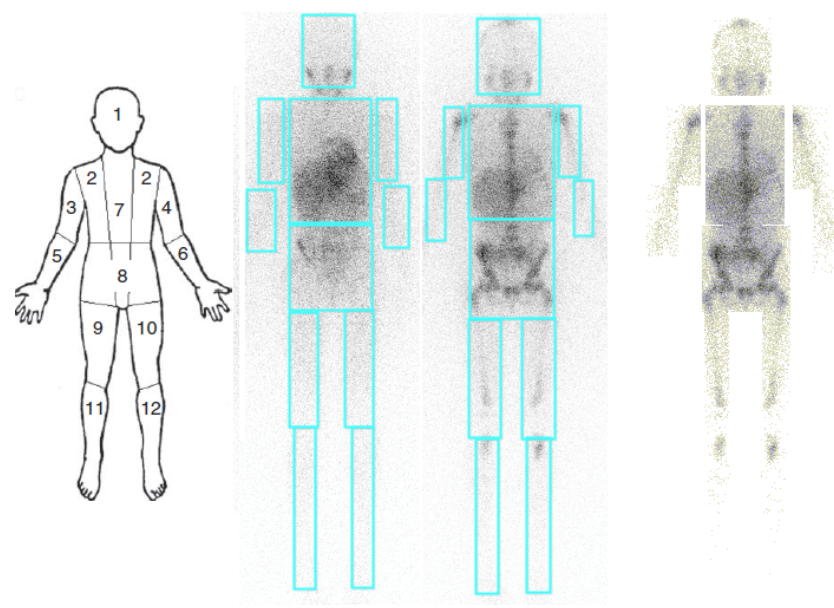

Fig. 1. SIOPEN semi-quantitative scoring method divides the skeleton into 12 anatomic segments as shown. On the central images, anterior views of patient $P A_{2}$ taken at pre chemotherapy time $T_{1}$, and at time $T_{2}$ after it, with the sectors of the anatomic division made for our anaylisis. On the right, image with only the body frame $I_{B F}$ created from the 12 sectors extracted from scan $T_{2}$.

The scan produces two planar images, the anterior and the posterior views. For every patient we have a scintigraphy in time $T_{1}$ pre chemotherapy and another one post it in time $T_{2}$. For some patients we have more scans, for example for patient $P A_{4}$, we have four successive scans $T_{1}$ to $T_{4}$. The group of ${ }^{123} \mathrm{I}-$ mIBG scans from each patient were interpreted by the nuclear medicine physicians of La Fe Hospital following current guidelines in (Bombardieri et al., 2010). 
This assessment is made in terms of description of the position of the lesions when they are visible on a given scan and, in consecutive scans of same patient, for each lesion previously detected, they include whether, in their appraisal, the lesion is more or less intense than in previous scans or if it is not visible any longer.

In planar image, the objective evidence of a tumour or metastasis is a peak of counts compared with local area counts average. For instance, tumours labeled as $\mathrm{ROI}_{1}, \mathrm{ROI}_{2}, \mathrm{ROI}_{3}$ and $\mathrm{ROI}_{4}$ in the series of scintigraphies shown in Fig. 2 are visible because of the high number of photons detected at their exact positions, greater than the average in the surroundings of tumours. Instead of using the surroundings of each tumour, that could be observer-dependent, we will test the use of each anatomical sector defined by the SIOPEN method as the predefined area for computing that local area counts average of reference for every ROI inside it.

In both views of every test scintigraphy, patient's body SIOPEN sectors (Lewington et al., 2009; 2011; Ladenstein et al., 2011) are identified in an image by placing a rectangle to delimit them: skull, thoracic cage, proximal right upper limb, distal right upper limb, proximal left upper limb, distal left upper limb, spine, pelvis, proximal right lower limb, distal right lower limb, proximal left lower limb and distal left lower limb as shown in Fig. 1. Then we split the original scintigraphy into 12 images identified as $I_{1}, I_{2}, \ldots, I_{12}$, one for each subimage of each sector in the original scan. We do that on both views.

We identify the image of the whole trunk as $I_{T}$ which is the union of SIOPEN Sectors 2 and 8 (see Figs. 1 and 2). We will use $I_{T}$ in order to facilitate taking measurements of uptake at the liver, where uptake is usually present, and to compare it with measurements of uptake at tumours or other ROIs in trunk. We also define $I_{B F}$ as the image of the body frame formed by extracting the subimages of all the SIOPEN sectors from original scintigraphy. $I_{B F}$ is a new image of the same size as the original scan which only contains patient's body sectors and the rest of the image is blank.

The liver and each tumour and metastatic region identified by phisicians of each patient are asigned to the SIOPEN sector where they are located. For each of them, we label regions as $\left\{\right.$ Liver, $\left.R O I_{1}, \ldots, R O I_{N_{i}}\right\}$, where $N_{i}$ is the number of ROIs at each specific patient $i, 1 \leq i \leq 10$.

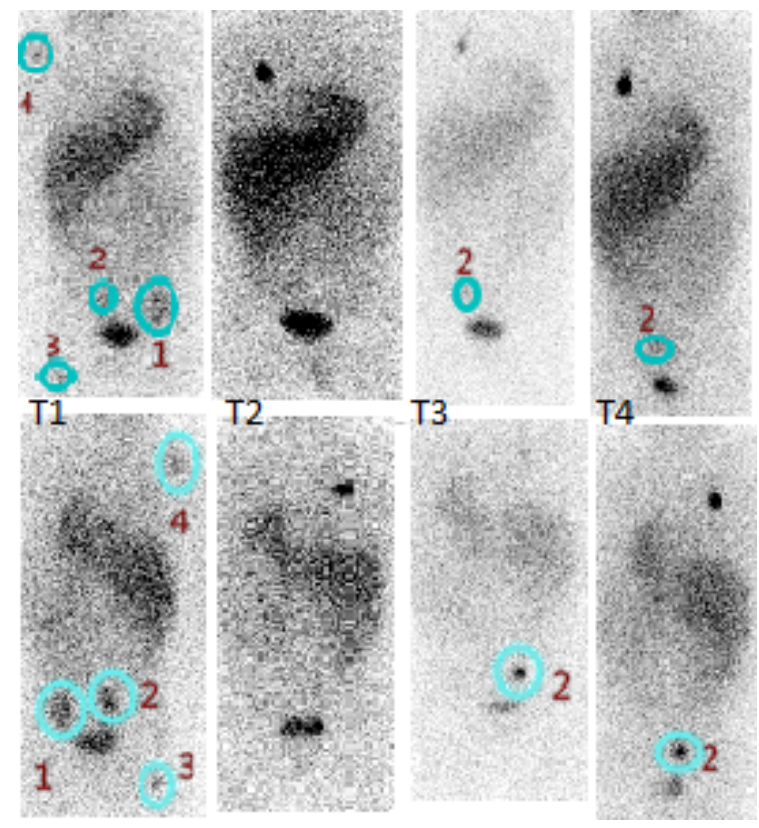

Fig. 2. $I_{T}$ sectors of scans of patient $\mathrm{PA}_{4}$ in time $T_{1}$ to $T_{4}$ with ROIs selected inside circles labeled from 1 to 4. On top, anterior $I_{T}$ views and on bottom the posterior ones.

Let $x_{i j}$ be the intensity (number of photon counts) at coordinates $(i, j)$ in any body sector $I_{k}$ with $k \in$ $\{1,2, \ldots, 12, T, B F\}$ that represents radionuclide uptake by tissue at these coordinates. We denote by $\bar{I}_{k}$ and $\sigma_{I_{k}}$ the average and standard deviation of $I_{k}$ respectively. In order to compare the count value $x_{i j}$ at pixel $(i, j) \in I_{k}$ with count value $x_{i j}^{\prime}$ at same patient's body coordinates in successive scintigraphies, we need both values transformed into the same uptake scale.

We will transform the number of photons counted at each pixel $x_{i j}$ into its $Z$-Score value $Z_{I_{k}}(i, j)$ in reference to its SIOPEN sector counts average $\bar{I}_{k}$ which will allow us to take objective and comparable uptake measurements,

$$
Z_{I_{k}}(i, j)=\frac{x_{i j}-\bar{I}_{k}}{\sigma_{I_{k}}}
$$

The brighter the pixels in the tumour are, the further they are above the average of sector $\bar{I}_{k}$. So our interest must focus in singling out those $x_{i j}$ values that are greater than $\bar{I}_{k}$ because they are very relevant uptake points. Consequently, we will use the transformation of Eq. 1 as in (Martínez-Díaz et al., 2013) but now applied to each SIOPEN sector:

$$
Q_{I_{k}}(i, j)=\max \left(Z_{I_{k}}(i, j), 0\right) .
$$

For the uptake measurement method to work properly, it is important to take into account only those pixels of image which unambiguously belong to the 
body frame, discarding pixels off the frame which have a value $x_{i j}$ whose origin is unknown (noise outside body frame).

For each image $I_{k}$, we denote by $\bar{Z}_{I_{k}}$ the average of $\left\{Z_{I_{k}}(i, j):(i, j) \in I_{k}\right\}$ and by $\bar{Q}_{I_{k}}$ the average of $\left\{Q_{I_{k}}(i, j):(i, j) \in I_{k}\right\} . Z_{I_{k}}$ transforms $I_{k}$ values into a new scale with zero mean and one as standard deviation. When applied to a data of a normal distribution, $\bar{Z}_{I_{k}}$ is defined by

$$
\frac{1}{\sqrt{2 \pi}} \int_{-\infty}^{\infty} x \mathrm{e}^{-x^{2} / 2} \mathrm{~d} x=0,
$$

and $\bar{Q}_{I_{k}}$, the average of the normalized values of those original values which were above the original average is a constant that could be calculated as

$$
\begin{aligned}
\frac{1}{\sqrt{2 \pi}} \int_{-\infty}^{\infty} u(x) x \mathrm{e}^{-x^{2} / 2} \mathrm{~d} x & =\frac{1}{\sqrt{2 \pi}} \int_{0}^{\infty} x \mathrm{e}^{-x^{2} / 2} \mathrm{~d} x \\
& =0.39894,
\end{aligned}
$$

where $u(x)$ is the Heaviside step function.

This means that, on average, the values that are above the mean have a normalized value of 0.39894 which we round to 0.40 . This value is a threshold of reference for the intensity. Any point with a value greater than of 0.40 has a value abnormally high in its sector.

With these prerequisites we are able to take objective measurements at the image of any region inside a SIOPEN sector and compare them with the next scintigraphies of the same patient. We will test the use of $\bar{Q}_{I_{k}}$ as the threshold of reference of specific uptake (tumour or metastases) at each SIOPEN sector and at $I_{T}$. We will test as well the use of $\bar{Z}_{I_{B F}}$ as a reference for comparing any region between different scintigraphies.

We are interested in taking measurements of the regions with specific uptake previously identified by physicians and of other predefined areas such as the liver. These measurements of the liver are used in semiquantitative scoring methods as a reference for the specific uptake.

In Martínez-Díaz et al. (2013), the next expression was introduced for measuring any $R O I_{\#}$ of the image. We adapt it for taking measurements in reference to the SIOPEN sector where the ROI is located:

$$
\bar{Q}_{I_{k}}\left(R O I_{\#}\right)=\frac{\sum\left\{Q_{I_{k}}(i, j):(i, j) \in R O I_{\#}\right\}}{C d\left(R_{H} O I_{\#}\right)},
$$

where $C d\left(R O I_{\#}\right)$ denotes the number of elements of $R O I_{\#}$ and $k \in\{1, \ldots, 12, T, B F\}$.
$\bar{Q}_{I_{k}}\left(R O I_{\#}\right)$ always takes positive values and it quantifies how higher $\mathrm{ROI}_{\#}$ is above the average of counts $\bar{I}_{k}$ of the SIOPEN sector $k$ where it is located.

In order to assist staff in taking an objective and repeatable measurement of a given $\mathrm{ROI}_{\#}$, at the moment of putting Eq. 3 into practice, it is important to unambiguously identify the exact points belonging to that $R O I_{\text {\#\# }}$.

As main evidence of tumour is the peak of counts, points unambiguosly belonging to it are bound to be closer to the maximum intensity at the body area where it is located.

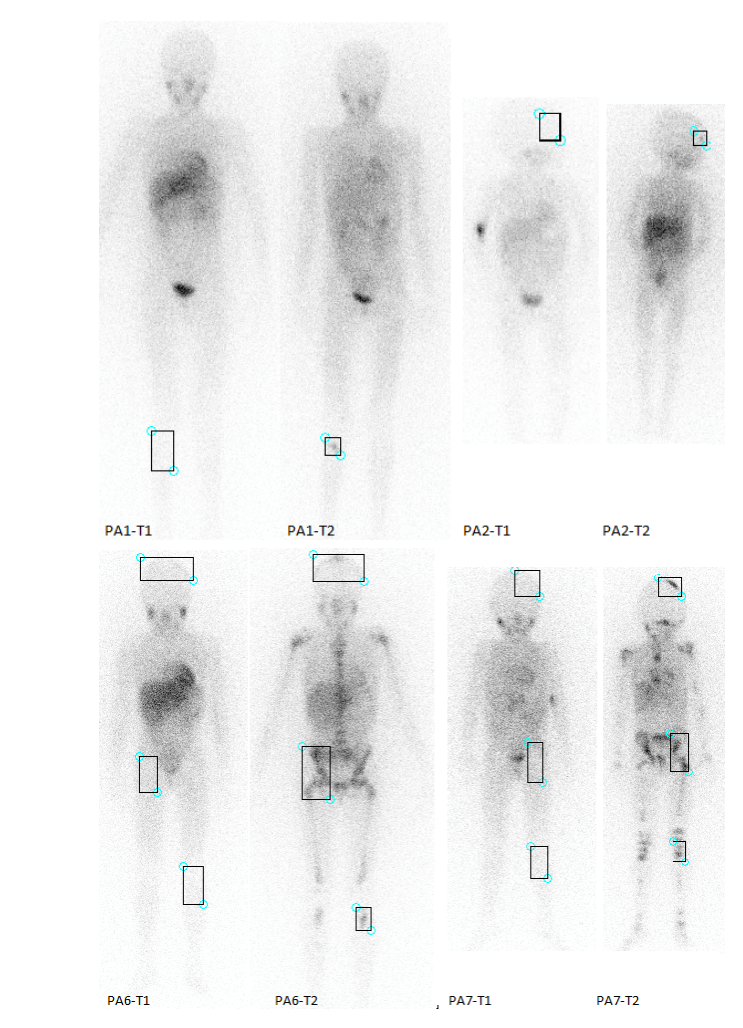

Fig. 3. Example of the process of taking quantitative measurements of any ROI in patients $P A_{1}, P A_{2}, P A_{6}$ and $P A_{7}$. Each ROI is demarcated inside a rectangle $\left(W_{R O I_{\#}}\right)$ close-fitted to it. Results are shown in Table 3.

Formally, assuming a given $R O I_{\#}$ is located within a SIOPEN sector $I_{k}$, we denote by $W_{R O I_{\#}}$ a rectangle around the whole $R O I_{\#}$, and only that $R O I_{\#}$, where it is included (see Fig. 3). This rectangle should be placed close-fitted to the shape of the $R O I_{\# \text { \#. If point }}$ at coordinates $(i, j) \in W_{R O I_{\#}}$ belongs to that ROI, then intensity $x_{i j} \in\left[X_{R O I t h r e s}, X_{R O I \max }\right]$ where $X_{\text {ROImax }}$ is the maximum value for intensity in $W_{R O I_{\#}}$ and $X_{R O I t h r e s}$ with $X_{\text {ROIthres }}<X_{R O I m a x}$ is the value of the minimum intensity for the $\mathrm{ROI}_{\text {\#. }}$. Consequently, we consider the following $\mathrm{ROI}_{\text {\# }}$ membership set:

$$
R O I_{\#}=\left\{\forall(i, j) \in W_{R O I_{\#}}: x_{i j} \in\left[X_{\text {ROIthres }}, X_{R O I m a x}\right]\right\} \text {. }
$$


The value of $X_{\text {ROIthres }}$ is unknown. As an approximation, we could consider it in reference to $X_{\text {ROImax }}$. This will lead us to a more observer independent measurement. For instance, we could fix $X_{\text {ROIthres }}=0.75 X_{\text {ROImax }}$. For simplicity, we will follow the criterion of selecting it equal to the minimum intensity in $W_{R O I_{\#}}$. In practice, this will lead to different measurements of the same $R O I_{\#}$ depending on the placement of $W_{R O I_{\#}}$ so that it should be close-fitted to the shape of it.

Besides measuring $\bar{Q}_{I_{k}}\left(R O I_{\#}\right)$, in our tests we also take measurements of the normalized $Z_{I_{k}}(i, j)$ values of the points belonging to the $R O I_{\#}$. We define $\bar{Z}_{I_{k}}\left(R O I_{\#}\right)$ as

$$
\bar{Z}_{I_{k}}\left(R O I_{\#}\right)=\frac{\sum\left\{Z_{I_{k}}(i, j):(i, j) \in R O I_{\#}\right\}}{C d\left(R O I_{\#}\right)},
$$

where $C d\left(R O I_{\#}\right)$ denotes the number of elements of the $R O I_{\#}$ and $k \in\{1, \ldots, 12, T, B F\}$.

$\bar{Z}_{I_{k}}\left(R O I_{\#}\right)$ takes positive and negative values. Negative measurements indicate that points in the selected $R O I_{\#}$ have lower values in average than $\bar{I}_{k}$. Positive measurements indicate the contrary.

In order to take objective measurements of any region of the body and to compare it to any other region located in any body part of the same or other scan, we should use the measurement related to the body frame $I_{B F}$, that is, $\bar{Z}_{I_{B F}}\left(R O I_{\#}\right)$.

Hence for every $R O I_{\#}$ we can take quantitative measurements with respect to the SIOPEN sector where it is located as well as to the whole body frame $I_{B F}$.

In case a tumour is visible in both views, it has two measurements, one referenced to $\bar{I}_{k}$ of the anterior view and the one referenced to the posterior view, as shown in Fig. 2.

\section{RESULTS}

Table 1 collects Sectors $\bar{Q}_{I_{k}}$ and $\bar{Z}_{I_{k}}$ example measurements in test patient $P A_{2}$ shown in Fig. 1. At both scans, $T_{1}$ pre-therapy and $T_{2}$ post it, reference values are similar at both views and close to the expected values 0.40 and 0.00 . Similar results were observed in the whole test set as shown in Fig. 4 where the trunk sectors $I_{T}$ of patients $P A_{2}, P A_{4}$ and $P A_{5}$ are shown as an example. Although the radiodistribution is very different in each patient, the values $\bar{Z}_{I_{T}}$ and $\bar{Q}_{I_{T}}$ are similar in every one as expected.

Table 2 shows a comparison between quantitative measurements and qualitative inform in patient $P A_{4}$
(Fig. 2). $\bar{Z}_{I_{T}}$ and $\bar{Z}_{I_{B F}}$ values are 0.00 in all the four $P A_{4}$ scans $T_{1}$ to $T_{4}$ although they presented variations in the intensity average $\bar{I}_{T}$. Each $\bar{Q}_{I_{T}}\left(R O I_{\#}\right)$ along the series of $\mathrm{PA}_{4}$ is an objective evidence of tumour uptake evolution. $\bar{Q}_{I_{T}}\left(R O I_{\#}\right)$ and $\bar{Z}_{I_{T}}\left(R O I_{\#}\right)$ measurements correlate well with qualitative ranks (visible or not, more or less intense, etc.) giving precise information of uptake change, that is, a positive increment when the peak is more intense and negative on the contrary. What is more, quantitative measurements $\bar{Q}_{I_{T}}\left(R O I_{\#}\right)$ give more intuitive information about the amount of uptake in tumours in reference to local sector counts average $\bar{I}_{T}$. Values of $\bar{Q}_{I_{T}}\left(R O I_{\#}\right)$ close to 0.00 imply light almost not visible uptake. Values from 0.00 to 0.40 mean higher but still light uptake in comparison to the count average $\bar{I}_{T}$. Finally, values of $\bar{Q}_{I_{T}}\left(R O I_{\#}\right)$ greater than 0.40 belong to a high uptake $R O I_{\#}$, abnormally higher than expected in sector, in comparison to $\bar{I}_{T}$.

Table 1. Example of $\bar{Q}_{I_{k}}$ and $\bar{Z}_{I_{k}}, k \in\{1,3, . ., 12, T, B F\}$ sector measurements in test patient $P A_{2}$ shown in Fig. 1. Succesive scans $T_{1}$, pre chemotherapy, and $T_{2}$, post it. Both reference values are similar, on both sides, and close to expected values 0.40 and 0.00 , respectively. Similar results were observed in the whole test set.

$P A_{2}$ measurements

\begin{tabular}{lcccc}
$P A_{2}$ & $T_{1}$ & & $T_{2}$ & \\
\hline & Anterior & Posterior & Anterior & Posterior \\
\hline $\bar{Q}_{I_{1}}$ & $\mathbf{0 . 3 9}$ & $\mathbf{0 . 3 8}$ & $\mathbf{0 . 3 8}$ & $\mathbf{0 . 3 9}$ \\
$\bar{Z}_{I_{1}}$ & -0.02 & -0.03 & -0.02 & -0.02 \\
\hline $\bar{Q}_{I_{T}}$ & $\mathbf{0 . 4 0}$ & $\mathbf{0 . 4 0}$ & $\mathbf{0 . 4 0}$ & $\mathbf{0 . 4 0}$ \\
$\bar{Z}_{I_{T}}$ & 0.00 & 0.00 & 0.00 & 0.00 \\
\hline $\bar{Q}_{I_{3}}$ & $\mathbf{0 . 4 0}$ & $\mathbf{0 . 3 9}$ & $\mathbf{0 . 3 9}$ & $\mathbf{0 . 4 0}$ \\
$\bar{Z}_{I_{3}}$ & 0.00 & 0.00 & 0.00 & 0.00 \\
\hline $\bar{Q}_{I_{4}}$ & $\mathbf{0 . 4 0}$ & $\mathbf{0 . 3 9}$ & $\mathbf{0 . 4 0}$ & $\mathbf{0 . 4 0}$ \\
$\bar{Z}_{I_{4}}$ & 0.00 & 0.00 & 0.00 & 0.00 \\
\hline $\bar{Q}_{I_{5}}$ & $\mathbf{0 . 4 0}$ & $\mathbf{0 . 3 6}$ & $\mathbf{0 . 3 9}$ & $\mathbf{0 . 4 0}$ \\
$\bar{Z}_{I_{5}}$ & 0.00 & 0.00 & 0.00 & 0.00 \\
\hline $\bar{Q}_{I_{6}}$ & $\mathbf{0 . 4 0}$ & $\mathbf{0 . 3 8}$ & $\mathbf{0 . 4 0}$ & $\mathbf{0 . 3 9}$ \\
$\bar{Z}_{I_{6}}$ & 0.00 & 0.00 & 0.00 & 0.00 \\
\hline $\bar{Q}_{I_{9}}$ & $\mathbf{0 . 3 8}$ & $\mathbf{0 . 3 9}$ & $\mathbf{0 . 4 0}$ & $\mathbf{0 . 4 0}$ \\
$\bar{Z}_{I_{9}}$ & 0.00 & 0.00 & 0.00 & 0.00 \\
\hline $\bar{Q}_{I_{10}}$ & $\mathbf{0 . 4 0}$ & $\mathbf{0 . 3 9}$ & $\mathbf{0 . 4 0}$ & $\mathbf{0 . 3 9}$ \\
$\bar{Z}_{I_{10}}$ & 0.00 & 0.00 & 0.00 & 0.00 \\
\hline $\bar{Q}_{I_{11}}$ & $\mathbf{0 . 3 3}$ & $\mathbf{0 . 3 5}$ & $\mathbf{0 . 3 3}$ & $\mathbf{0 . 3 2}$ \\
$\bar{Z}_{I_{11}}$ & 0.00 & 0.00 & 0.00 & 0.00 \\
\hline $\bar{Q}_{I_{12}}$ & $\mathbf{0 . 3 3}$ & $\mathbf{0 . 3 5}$ & $\mathbf{0 . 3 8}$ & $\mathbf{0 . 3 5}$ \\
$\bar{Z}_{I_{12}}$ & 0.00 & 0.00 & 0.00 & 0.00 \\
\hline $\bar{Q}_{I_{B F}}$ & $\mathbf{0 . 3 9}$ & $\mathbf{0 . 4 0}$ & $\mathbf{0 . 3 6}$ & $\mathbf{0 . 3 9}$ \\
$\bar{Z}_{I_{B F}}$ & 0.00 & 0.00 & 0.00 & 0.00 \\
\hline & & & & \\
\hline
\end{tabular}




\section{DISCUSSION}

One of the big problems of all the existing mIBG scores is the subjetivity of the interpretation of the scans that makes very difficult the quantitation of the response to treatment. Our series of patients, can be considered as a pilot demonstration of the possibility of truly quantify the extension of disease at diagnosis and the response to treatment, avoiding inter-observer variations.

In every analyzed ${ }^{123} \mathrm{I}-\mathrm{mIBG}$ of test set, the mean of the standard Z-Score $\bar{Z}_{I_{k}}$ for any SIOPEN body sector $I_{k}$ as well as $\bar{Z}_{I_{B F}}$ tends to be constant with value close to 0.0. This fact enables us to take comparable measurements with respect to the local counts average at any SIOPEN sector $\bar{I}_{k}$. $\bar{Z}_{I_{k}}$ and $\bar{Z}_{I_{B F}}$ equal to 0.00 is the common point of reference between scans of the same patient, and even between different patients. Regional comparisons to the predefined SIOPEN sectors rather than to local lesion site reduces observer dependent measurements.

In the same patient and the same scan, comparisons between measurements $\bar{Z}_{I_{k}}\left(R O I_{\#}\right)$ taken at ROIs of same sector $I_{k}$ are direct. For instance, objective comparison between the specific uptake $\bar{Z}_{I_{k}}\left(R O I_{\#}\right)$ due to a possible tumour and non-specific uptake at liver $\bar{Z}_{I_{k}}$ (Liver) is possible.

Comparisons between ROIs located in different SIOPEN sectors by using $\bar{Z}_{I_{k}}\left(R O I_{\#}\right)$ and $\bar{Z}_{I_{k^{\prime}}}\left(R O I_{I^{\prime}}\right)$ could be deceptive in such cases where one of the sectors has an abnormal counts increase, for instance, due to a whole sector ${ }^{123} \mathrm{I}-\mathrm{mIBG}$ stain in skin.

Table 2. Comparison between qualitative informs (visible or not, more or less intense, etc.) and quantitative meassurements of tumours in posterior views of scans $T_{1}$ and $T_{2}$ (quantitative difference in parentheses) of patient $P_{4}$ (Fig. 2). $\bar{Z}_{I_{B F}}$ and $\bar{Z}_{I_{T}}$ are 0.0 at every scintigraphy. Both references enable to take quantitative comparisons of uptake between scintigraphies. $\bar{Q}_{I_{B F}}$ and $\bar{Q}_{I_{T}}$ are close to 0.40 at each scan as expected. This is a reference for comparisons to local sector average. Values of $\bar{Q}_{I_{T}}\left(R O I_{\#}\right)$ higher than $0.40\left(\bar{Q}_{I_{T}}\right)$ means that the ROI\# has an abnormal value of counts, higher than the average of the trunk sector $I_{T}$.

\begin{tabular}{|c|c|c|c|c|}
\hline \multicolumn{5}{|c|}{$P A_{4}$ measurements } \\
\hline$P A_{4}$ & $T_{1}$ & $T_{2}$ & $T_{3}$ & $T_{4}$ \\
\hline$\overline{\bar{I}_{T}}$ & 0.29 & 0.40 & 0.16 & 0.29 \\
\hline $\bar{Z}_{I_{T}}$ & 0.00 & 0.00 & 0.00 & 0.00 \\
\hline $\bar{Q}_{I_{T}}$ & 0.39 & 0.40 & 0.38 & 0.39 \\
\hline $\bar{Z}_{I_{B F}}$ & 0.00 & 0.00 & 0.00 & 0.00 \\
\hline$\overline{\bar{Q}_{I_{T}}(\text { Liver })}$ & 0.92 & 0.95 & 0.78 & 1.09 \\
\hline $\bar{Z}_{I_{T}}($ Liver $)$ & 0.84 & 0.86 & 0.67 & 1.05 \\
\hline $\bar{Z}_{I_{B F}}($ Liver $)$ & 1.59 & 1.59 & 1.41 & 1.84 \\
\hline Qualitative & Visible & Not visible & Not Visible & Not Visible \\
\hline $\bar{Q}_{I_{T}}\left(R O I_{1}\right)$ & 0.40 & $0.05(-0.35)$ & $0.03(-0.02)$ & $0.00(-0.03)$ \\
\hline $\bar{Z}_{I_{T}}\left(R O I_{1}\right)$ & 0.11 & $-0.56(-0.67)$ & $-0.62(-0.06)$ & $-0.71(-0.09)$ \\
\hline $\bar{Z}_{I_{B F}}\left(R O I_{1}\right)$ & 0.76 & $0.00(-0.76)$ & $-0.07(-0.07)$ & $-0.16(-0.09)$ \\
\hline Qualitative & Visible & Not visible & More Intense & Less Intense \\
\hline $\bar{Q}_{I_{T}}\left(R O I_{2}\right)$ & 0.38 & $0.23(-0.15)$ & $0.90(+0.67)$ & $0.73(-0.17)$ \\
\hline $\bar{Z}_{I_{T}}\left(R O I_{2}\right)$ & 0.19 & $0.01(-0.18)$ & $0.72(+0.71)$ & $0.66(-0.06)$ \\
\hline $\bar{Z}_{I_{B F}}\left(R O I_{2}\right)$ & 0.85 & $0.64(-0.21)$ & $1.23(+0.59)$ & $1.12(-0.11)$ \\
\hline Qualitative & Visible & Not visible & Not Visible & Not Visible \\
\hline $\bar{Q}_{I_{T}}\left(R I_{3}\right)$ & 0.05 & $0.00(-0.05)$ & $0.00(\approx)$ & $0.00(\approx)$ \\
\hline $\bar{Z}_{I_{T}}\left(R O I_{3}\right)$ & -0.61 & $-0.79(-0.18)$ & $-0.87(-0.08)$ & $-0.81(+0.06)$ \\
\hline $\bar{Z}_{I_{B F}}\left(\mathrm{ROI}_{3}\right)$ & -0.06 & $-0.26(-0.20)$ & $-0.35(-0.09)$ & $-0.27(+0.08)$ \\
\hline Qualitative & Visible & Not visible & Not Visible & Not Visible \\
\hline $\bar{Q}_{I_{T}}\left(R \mathrm{I}_{4}\right)$ & 0.06 & $0.00(-0.06)$ & $0.02(+0.02)$ & $0.02(\approx)$ \\
\hline $\bar{Z}_{I_{T}}\left(R O I_{4}\right)$ & -0.47 & $-0.68(-0.21)$ & $-0.67(+0.01)$ & $-0.63(+0.04)$ \\
\hline $\bar{Z}_{I_{B F}}\left(R^{\prime} I_{4}\right)$ & 0.10 & $-0.14(-0.24)$ & $-0.13(+0.01)$ & $-0.06(+0.07)$ \\
\hline
\end{tabular}




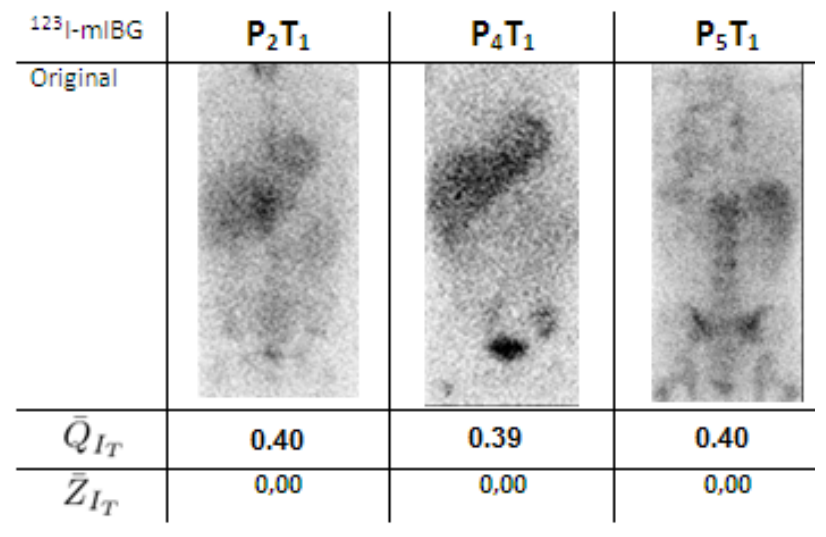

Fig. 4. Example of sector $I_{T}$ measurements in patients $P A_{2}, P A_{4}$ and $P A_{5}$. Although the radiodistribution is very different in each patient, the value of $\bar{Z}_{I_{T}}$ is close to 0.00 and $\bar{Q}_{I_{T}}$ is close to 0.40 in all of them. Same results were observed in the whole test set.

In order to properly compare ROIs at different sectors, the value of $\bar{Z}_{I_{B F}}\left(R O I_{\#}\right)$ of each ROI may be used. The whole body counts average $\bar{I}_{B F}$ is used as the numeric reference for taking quantitative measurements in the same way that it is already a visual implicit reference for the human eye for the qualitative assessment.

What is more, comparisons between non-specific background uptake at any SIOPEN sector $\bar{Z}_{I_{B F}}\left(I_{k}\right)$ with respect to the whole body frame $\bar{Z}_{I_{B F}}$ are also possible. At limb sectors, for instance at Sector 9, $\bar{Z}_{I_{B F}}\left(I_{9}\right)$ measurement should be low in patients with no metastatic sites at that sector. The increase of this objective metric is an evidence of an abnormal uptake at this sector with respect to the whole body, that is, a possible metastatic site in bones.

This fact makes $\bar{Z}_{I_{B F}}\left(R O I_{\#}\right)$ a good candidate to take standarized comparable measurements between different patients and different scans. A database of usual $\bar{Z}_{I_{B F}}\left(R O I_{\#}\right)$ values of tumour and metastases at each SIOPEN sector could be used for comparisons in cases of doubt.

Quantitative measurements $\bar{Q}_{I_{k}}\left(R O I_{\#}\right)$ and the fact that $\bar{Q}_{I_{k}}$ is always close to 0.40 gives an intuitive visual reference value of how intense is that ROI in reference to the local counts average $\bar{I}_{k}$.

The use of $\bar{Q}_{I_{B F}}\left(R O I_{\#}\right)$ could be problematic because it takes only positive values and it is related to $\bar{I}_{B F}$. It is possible for a given ROI to be greater than the average at its sector but lower than $\bar{I}_{B F}$ and then $\bar{Q}_{I_{B F}}\left(R O I_{\#}\right)$ would be 0.0 . In such cases, the value of $\bar{Z}_{I_{B F}}\left(R O I_{\#}\right)$ would be negative meaning that the ROI has a counts average lower than the average at the whole body frame $I_{B F}$.
These results show that the use of $\bar{Q}_{I_{k}}\left(R O I_{\#}\right)$ and $\bar{Q}_{I_{B F}}\left(R O I_{\#}\right)$ could be useful for automatic detection of abnormal uptake regions on image. If any region in body has a value greater than $\bar{Q}_{I_{B F}}$ then it is bound to be abnormal, because it is much too intense. $\bar{Q}_{I_{k}}\left(R O I_{\#}\right)$ could be used in the same manner.

Table 3. Results of measurements in anterior views of scans of patients $P A_{2}$ and $P A_{6}$ in Fig. 3. Comparisons between different patients are possible by using $\bar{Z}_{I_{B F}}\left(R_{O} I_{\#}\right)$ because it is referenced to body frame counts average $\bar{I}_{B F}$ and $\bar{Z}_{I_{B F}}$ has a value of 0.0 in every scan of every patient.

\begin{tabular}{|c|c|c|}
\hline \multicolumn{3}{|c|}{$P A_{2}$ and $P A_{6}$ measurements } \\
\hline$P A_{2}$ & $T_{1}$ & $T_{2}$ \\
\hline$\overline{\bar{Z}_{I_{1}}}$ & 0.00 & 0.00 \\
\hline $\bar{Z}_{I_{B F}}$ & 0.00 & 0.00 \\
\hline Qualitative & Not visible & Visible \\
\hline $\bar{Q}_{I_{1}}\left(R O I_{1}\right)$ & 0.05 & $0.14(+0.09)$ \\
\hline $\bar{Z}_{I_{1}}\left(R O I_{1}\right)$ & -0.53 & $-0.43(+0.10)$ \\
\hline $\bar{Z}_{I_{B F}}\left(R O I_{1}\right)$ & -0.56 & $-0.52(+0.04)$ \\
\hline Qualitative & Not visible & Visible \\
\hline $\bar{Q}_{I_{T}}\left(R O I_{2}\right)$ & 0.00 & $0.53(+0.53)$ \\
\hline $\bar{Z}_{I_{T}}\left(R O I_{2}\right)$ & -0.84 & $0.15(+0.91)$ \\
\hline $\bar{Z}_{I_{B F}}\left(R O I_{2}\right)$ & -0.30 & $1.01(+1.31)$ \\
\hline Qualitative & Not visible & Visible \\
\hline $\bar{Q}_{I_{12}}\left(\mathrm{ROI}_{3}\right)$ & 0.64 & $0.90(+0.26)$ \\
\hline $\bar{Z}_{I_{12}}\left(R O I_{3}\right)$ & 0.39 & $0.75(+0.36)$ \\
\hline $\bar{Z}_{I_{B F}}\left(R O I_{3}\right)$ & -0.80 & $-0.57(+0.23)$ \\
\hline$P A_{6}$ & $T_{1}$ & $T_{2}$ \\
\hline$\overline{Z_{I_{1}}}$ & 0.00 & 0.00 \\
\hline $\bar{Z}_{I_{B F}}$ & 0.00 & 0.00 \\
\hline Qualitative & Not visible & Visible \\
\hline $\bar{Q}_{I_{1}}\left(R O I_{1}\right)$ & 0.07 & $0.50(+0.43)$ \\
\hline $\bar{Z}_{I_{1}}\left(R O I_{1}\right)$ & -0.42 & $0.07(+0.49)$ \\
\hline $\bar{Z}_{I_{B F}}\left(R O I_{1}\right)$ & -0.44 & $-0.16(+0.38)$ \\
\hline Qualitative & Not visible & Visible \\
\hline $\bar{Q}_{I_{T}}(R$ & 0.08 & $0.59(+0.51)$ \\
\hline $\bar{Z}_{I_{T}}\left(R O I_{2}\right)$ & -0.63 & $0.22(+0.85)$ \\
\hline $\bar{Z}_{I_{B F}}\left(R O I_{2}\right)$ & 0.13 & $0.98(+0.85)$ \\
\hline Qualitative & Not visible & Visible \\
\hline $\bar{Q}_{I_{12}}\left(R O I_{3}\right)$ & 0.51 & $0.94(+0.43)$ \\
\hline $\bar{Z}_{I_{12}}\left(R O I_{3}\right)$ & 0.19 & $0.79(+0.80)$ \\
\hline $\bar{Z}_{I_{B F}}\left(R O I_{3}\right)$ & -0.95 & $-0.25(+0.70)$ \\
\hline
\end{tabular}




\section{CONCLUSIONS}

In this paper we propose the application of a method to take objective observer independent measurement of the specific ${ }^{123} \mathrm{I}$-mIBG uptake at any region of interest in reference to the SIOPEN sector where it is located.

When response to treatment is light, low definition in scan may make impossible to the human eye to appreciate changes in the intensity. $\bar{Z}_{I_{B F}}\left(R O I_{\#}\right)$, $\bar{Z}_{I_{k}}\left(R O I_{\#}\right)$ and $\bar{Q}_{I_{k}}\left(R O I_{\#}\right)$ measurements may assist in the application of SIOPEN validated semi-quantitative scoring method as an additional tool for measuring and comparing ${ }^{123} \mathrm{I}$-mIBG uptake in doubt cases in which differences in assessment between observers could appear.

$\bar{Z}_{I_{B F}}\left(R O I_{\#}\right)$ is a measuring tool for absolute comparison between any regions and between patients and scans. It has an intuitive measurement of how much a region of interest stands out among the rest of the body. It enables to take objective measurements of uptake in a region in reference to the whole body frame counts average to precise the increase or decrease of uptake in that region in comparison with previous scans of the same or other patient.

$\bar{Q}_{I_{k}}\left(R O I_{\#}\right)$ and $\bar{Z}_{I_{k}}\left(R O I_{\#}\right)$ enable the objective comparison of the specific uptake measurements at different ROIs inside the same SIOPEN sector. They are referenced to the local counts average at SIOPEN sector $k . \bar{Q}_{I_{k}}\left(R O I_{\#}\right)$ has shown an intuitive interpretation. It is close to 0.00 when the uptake at region is hardly visible. As uptake increases, the peak is more visible and $\bar{Q}_{I_{k}}\left(R O I_{\#}\right)$ increases accordingly taking values lower than 0.40 when is not intense and higher than 0.40 when it is a very intense peak. At limbs, the significance of $\bar{Q}_{I_{k}}\left(R O I_{\#}\right)$ is slightly different, that is, values close to 0.40 of any ROI are due to peaks more intense (and abnormal) at trunk than in limbs, where uptake peaks reach higher $\bar{Q}_{I_{k}}\left(R O I_{\#}\right)$ values.

$\bar{Q}_{I_{k}}\left(R O I_{\#}\right)$ greater than 0.40 is a good candidate to automatically detect abnormal uptake in head and trunk sectors. That is, any region in sector with $\bar{Q}_{I_{k}}\left(R O I_{\#}\right)$ greater than 0.40 has a high abnormal uptake value and is very likely to be an abnormal uptake peak. But, in limb sectors, due to the low counts average, there are always regions with values greater than 0.40 although they are not visible (they are not abnormal peaks).

In such sectors, for automatic detection, the values of $\bar{Z}_{I_{B F}}\left(R O I_{\#}\right)$ should be used because it is referenced to whole body counts average $\bar{I}_{B F}$. At any sector, a region with a value $\bar{Q}_{I_{B F}}\left(R O I_{\#}\right)$ greater than 0.40 could be automatically identified as abnormal.

\section{ACKNOWLEDGMENTS}

This work has been supported by "Ayudas para Actividades Preparatorias de Proyectos Coordinados entre Investigadores de la Universitat Politècnica de València e Investigadores del Hospital Universitario y Politécnico La Fe", II Call, 2013, AUTOSCOREMIBG project. The authors are very grateful to the referees for their useful comments.

\section{REFERENCES}

Ady N, Zucker J, Asselain B, Edeline V, Bonnin F, Michon J, Gongora R, Manil L (1995). A new 123i-mibg whole body scan scoring method-application to the prediction of the response of metastases to induction chemotherapy in stage iv neuroblastoma. Eur J Cancer 31A:256-61.

Bombardieri E, Giammarile F, Aktolun C, Baum R, Delaloye A, Maffioli L, et al. (2010). 131i/123imetaiodobenzylguanidine (mibg) scintigraphy procedures guidelines for tumour imaging. Eur $\mathbf{J}$ Nucl Med Mol I 37:2436-46.

Brisse HJ, McCarville MB, Granata C, Krug KB, WoottonGorges SL, Kanegawa K, et al. (2011). Guidelines for imaging and staging of neuroblastic tumors: Consensus report from the international neuroblastoma risk group project. Radiology 261:243-57.

Bulten B, van der Palen R, van Laarhoven H, Kapusta L, Mavinkurve-Groothuis A, de Geus-Oei L (2012). Interobserver variability of heart-to-mediastinum ratio in i-123 mibg sympathetic imaging. Eur J Nucl Med Mol I 39:S508.

Charron M (2013). Contemporary approach to diagnosis and treatment of neuroblastoma. Q J Nucl Med Mol Im 57:40-52.

Chen W, Cao Q, Dilsizian V (2011). Variation of heartto-mediastinal ratio in (123)i-mibg cardiac sympathetic imaging: its affecting factors and potential corrections. Curr Cardiol Rep 13:132-7.

Decarolis B, Scheneider C, Hero B, Simon T, Volland R, Roels F, et al. (2013). Iodine-123 metaiodobenzylguanidine scintigraphy scoring allows prediction of outcome in patients with stage 4 neuroblastoma: Results of the cologne interscore comparison study. J Clin Oncol 31:944-51.

Frappaz D, Bonneu A, Chauvot P, Edeline V, Giammarile F, Siles S, et al. (2000). Metaiodobenzylguanidine assessment of metastatic neuroblastoma: observer dependency and chemosensitivity evaluation. the sfop group. Med Pediatr Oncol 34:237-41.

Hannequin P, Mas J (2002). Statistical and heuristic image noise extraction (shine): a new method for processing 
poisson noise in scintigraphic images. Phys Med Biol 47:4329-44.

Inoue Y, Abe Y, Itoh Y, Asano Y, Kikuchi K, Sakamoto $\mathrm{Y}$, et al. (2013). Acquisition protocols and correction methods for estimation of the heart-to-mediastinum ratio in 123i-metaiodobenzylguanidine cardiac sympathetic imaging. J Nucl Med 54:707-13.

Jeong CB, Kim KG, Kim TS, Kim SK (2011). Comparison of image enhancement methods for the effective diagnosis in successive whole-body bone scans. J Digit Imaging 24:424-36.

Jia-Yann H, Pan-Fu K, Yung-Sheng C (2007). A set of image processing algorithms for computer-aided diagnosis in nuclear medicine whole body bone scan images. IEEE T Nucl Sci 54:514-22.

Krom AJ, Wickham F, Hall ML (2013). Evaluation of image enhancement software as a method of performing halfcount bone scans. Nucl Med Commun 34:78-85.

Ladenstein R, Poetschger U, Boubaker A, Bar-Sever Z, Drake B, Staudenherz A, et al. (2011). The prognostic value of semi-quantitative I-123 MIBG scintigraphy at diagnosis in high risk neuroblastoma: validation of the SIOPEN score method. Pediatr Blood Cancer 57:7323.

Lewington V, Poetschger U, Boubaker A, Bar-Sever Z, Drake B, Staudenherz A, et al. (2011). The prognostic value of semi-quantitative I-123 MIBG scintigraphy at diagnosis in high-risk neuroblastoma: Validation of the SIOPEN score method. J Clin Oncol 29:(suppl; abstract 9511).

Lewington V, Sever ZB, Lynch T, Giammarile F, McEwan A, Shulkin B, et al. (2009). Development of a new, semiquantitative I-123 MIBG reporting method in high risk neuroblastoma. Eur J Nucl Med Mol I 50:1379.

Maisey M, Natarajan T, Hurley P, Jr HW (1973). Validation of a rapid computerized method of measuring 99mtc pertechnetate uptake for routine assessment of thyroid structure and function. J Clin Endocrinol Metab 36:317-22.
Martínez-Díaz R, Balaguer J, Sánchez-Ruiz LM, Bello P, Castel V, Perís-Fajarnés G (2013). On analytical methods in neuroblastoma detection. Abstr Appl Anal 2013:341346.

Matthay K, Shulkin B, Ladenstein R, Michon J, Giammarile $\mathrm{F}$, Lewington V, et al. (2010). Criteria for evaluation of disease extent by 123i-metaiodobenzylguanidine scans in neuroblastoma: a report for the international neuroblastoma risk group (inrg) task force. Brit J Cancer 102:1319-26.

Messina J, Cheng S, Franc B, Charron M, Shulkin B, To $\mathrm{B}$, et al. (2006). Evaluation of semiquantitative scoring system for metaiodobenzylguanidine (mibg) scans in patients with relapsed neuroblastoma. Pediatr Blood Cancer 47:865-74.

Mueller W, Coppenrath E, Pfluger T (2013). Nuclear medicine and multimodality imaging of pediatric neuroblastoma. Pediatr Radiol 43:418-27.

Sajn L, Kukar M, Kononenko I, Milcinski M (2005). Computerized segmentation of whole-body bone scintigrams and its use in automated diagnostics. Comput Meth Prog Bio 80:47-55.

Sharp S, Parisi M, Gelfand M, Yanik G, Shulkin B (2013). Functional-metabolic imaging of neuroblastoma. Q J Nucl Med Mol Im 57:6-20.

Wesolowski C, Yahil A, Puetter R, Babyn P, Gilday D, Khan M (2005). Improved lesion detection from spatially adaptive, minimally complex, pixon reconstruction of planar scintigraphic images. Comput Med Imag Grap 29:65-81.

Yanik GA, Parisi MT, Shulking BL, Naranjo A, Kreissman SG, London WB, et al. (2013). Semiquantitative mibg scoring as a prognostic indicator in patients with stage 4 neuroblastoma: A report from the children's oncology group. J Nucl Med 54:541-8.

Yin T, Chiu N (2004). A computer-aided diagnosis for locating abnormalities in bone scintigraphy by a fuzzy system with a three-step minimization approach. IEEE T Med Imaging 23:639-54. 